\title{
Reliability Analysis of a Two Nonidentical Unit Parallel System with Optional Vacations under Poisson Shocks
}

\author{
Mohamed S. El-Sherbeny $\mathbb{D}^{1,2}$ and Zienab M. Hussien ${ }^{1}{ }^{1}$ \\ ${ }^{1}$ Department of Mathematics, Faculty of Sciences and Arts-Rabigh Campus, King Abdulaziz University, Jeddah, Saudi Arabia \\ ${ }^{2}$ Department of Mathematics, Faculty of Science, Helwan University, Cairo, Egypt \\ Correspondence should be addressed to Mohamed S. El-Sherbeny; m_el_sherbeny@yahoo.com
}

Received 2 January 2022; Revised 22 January 2022; Accepted 25 January 2022; Published 23 February 2022

Academic Editor: Gianpaolo Di Bona

Copyright (c) 2022 Mohamed S. El-Sherbeny and Zienab M. Hussien. This is an open access article distributed under the Creative Commons Attribution License, which permits unrestricted use, distribution, and reproduction in any medium, provided the original work is properly cited.

\begin{abstract}
In this article, we study the impact of some system parameters on an industrial system consisting of two nonidentical parallel units with one repairer. The active unit may fail due to essential factors such as aging or deterioration, or due to external phenomena such as Poisson shocks occurring at different time periods. Whenever the value of a shock is greater than the specified threshold of the active unit, the active unit fails. The article assumes that the repairman can make one of two decisions at the beginning of system operation: either he takes a vacation when the two units are operating normally, or he remains in the system to monitor it until the first failure of the system. If a failure occurs in either unit during the repairman's absence, the failed unit must wait until the repairman is called back to work. We assume that the value of each shock is i.i.d. with a known distribution. The length of the repairman's vacation, the repair time, and the recall time are arbitrary distributions. Various reliability measures were calculated using the supplementary variable technique and the theory of Markov vector processes. Sensitivity and relative sensitivity analyses were also performed for the system parameters. Finally, numerical calculations and graphical analyses were performed to validate the derived indices.
\end{abstract}

\section{Introduction}

The economic progress of any country depends to a great extent on the development of the industrial and mechanical fields. This progress leads to the emergence of new technical skills that help in solving problems that may arise in these complex industrial systems. However, despite all these kinds of help, consumers want cost-effective and highly efficient industrial systems. Therefore, system designers and researchers in this field face a great challenge to develop many systems and increase their efficiency, reliability, and safety.

In the past, many research studies have been done to explain the concept of reliability, to increase the efficiency of many industrial systems, and to analyze the cost of different redundant systems under the effect of some constraints such as the periods of time when the system is on or off, the different types of faults affecting this system, the different types of repair of these units, the different types of leave for repairmen, etc. Reliability measures such as mean time to failure are effective measures when analyzing engineering models. The applications of reliability were introduced in many papers in literature (see, for example, [1]). Lin et al. [2] explained that the maintenance of an offshore wind turbine accounts for more than $30 \%$ of the total cost of its life cycle. According to Ren et al. [3], preventive maintenance based on a predetermined period is considered as a more advanced approach. Optimizing the selection of the specified interval can help, in turn, to optimize the preventive maintenance strategies. Preventive maintenance brings various advantages such as avoiding unplanned maintenance, combining maintenance and repairs, and optimizing maintenance tasks.

Most research in the past focused on three types of classical random shock models: cumulative shock models, extreme shock models, and $\delta$-shock models. In this context, we present some research papers that deserve to be mentioned. For example, Lam and Zhang [4] studied a shock model for the maintenance problem of a repairable system. $\mathrm{Li}$ and Zhao [5] presented complex systems containing $n$ 
i.i.d. units with the $\delta$-shock model. Recently, El-Sherbeny [6] studied the behavior of the repairable cold standby system under the effect of Poisson shocks. In discussing the above research, we have found that the failed device is repaired immediately, but, in reality, the failed device may not be repaired immediately for various reasons. In discussing the above researches, we have found that the failed device is repaired immediately, but in reality, the failed device may not be repaired immediately because the multiple leaves for the technician or he is busy repairing another device. This happens in medium or small factories because they may not be able to afford to hire a full-time repair technician to take care of their equipment. Instead, a technician may have to take care of many types of equipment, and he may not be able to repair a failed piece of equipment as soon as it fails. For this reason, the reliability theory has been studied with vacation [7-13]. Therefore, the vacation concept was first presented in the model analysis of queuing systems in [14], and the types of vacation policies are defined as follows: single vacation and multiple vacations. For a comprehensive and excellent study of the latest results for a variety of leave models, including some applications [15], Ke and Wang [16] studied a machine repair problem with different vacation policies, both based on a queuing theory viewpoint.

In the current research, the deterioration to the system from the first shock can be neglected, and the system fails once the shocks accumulate to a certain level. However, the concept of the policy of optional leave of repairers with the effect of Poisson shocks for any repairable system has not appeared in the previous literature. Therefore, in light of the above observations, we have analyzed here a repairable system with optional vacations for a repairman in starting the system work under the effect of Poisson shocks on active units using the supplementary variables technique. Suppose that the system could be subjected to shocks that follow a Poisson process, and the technician has the choice of either staying in the system to monitor the two units until one fails or taking leave when the two units are operating normally. Note that analyzing the behavior of the system is not a simple task when most of the time the system follows the general distributions. The method of supplementary variable helps to solve the partial differential equations associated with the description of the dynamics of motion between the different states of the system. Using the ergodicity of the investigated process and the method of supplementary variables, we obtain explicit expressions of the reliability metrics such as reliability function, steady-state availabilities, mean time to system failure, the steady-state probability that the repairman is on vacation, the steady-state probability that the system is waiting for repair, and steady-state failure frequency.

This article is designed as follows: Section 2 introduces more details about the system and assumptions of the system description. In Sections 3 and 4, the state equations of the system are constructed to derive the steady-state availability before first failure and the mean time to first failure using the supplementary variable technique method. The numerical examples are presented in Section 5. In Section 6, sensitivity and relative sensitivity analyses of the reliability characteristics are performed. The conclusions are summarized in the last section.

\section{Description of the System and Assumptions}

The system which consists of two nonidentical units and a single repairman under Poisson shock is subjected to the following assumptions:

(i) A1: in the initial period, both devices operate at high efficiency, and a repairman has the choice of either staying in the system or taking a vacation.

(ii) A2: the system is exposed to shocks continually. The arrival of shocks is considered as a Poisson process $\{S(t), t \geq 0\}$ with strength $\lambda_{i}>0$. The magnitude of each shock $\hat{Y}$, independent random variable with distribution function $F$.

(iii) A3: when a shock occurs, it affects the active units (A, B). The active units will fail when the magnitude of a shock exceeds a threshold. The threshold of units is a non-negative random variable $\tau_{i}$ with a distribution function $\xi_{i}$.

(iv) A4: when any unit fails with the existence of the repairman in the system, it will be repaired immediately. Once the repairman is done repairing the failed units, the repairman has the choice to either stay at the system or take a vacation, and then, he returns from vacation if at least one unit is failing. The repair rule is "first-in-first-out". If a unit fails while the other is being repaired, the recently failed unit must wait for repair, and the system has to stop working.

(v) A5: shocks are the main reason for units to fail, and the system fails only if both the units fail.

Based on the preceding assumptions, we can conclude that the failure probability of units, given the shock value $\hat{y}$, $\xi_{i}(\hat{y})=P\left(\tau_{i} \leq \hat{y}\right)$. Since the magnitude of a shock is a random variable $\hat{Y}$, the conditional failure probability of units is a random variable $\xi_{i}(\widehat{Y}) \quad(i=1,2)$, and their probability distribution can be written by

$$
\begin{aligned}
P_{i}(y) & =P\left(\xi_{i}(\widehat{Y}) \leq y\right) \\
& =P\left(\widehat{Y} \leq \xi_{i}^{-1}(y)\right) \\
& =F\left(\xi_{i}^{-1}(y)\right), \quad 0 \leq y \leq 1,(i=1,2) .
\end{aligned}
$$

According to the above assumptions $A_{2}$ and $A_{3}$, the probability that single shock causes unit (A or $B$ ) to fail is

$$
\begin{aligned}
r_{i}=P\left(\widehat{Y}>\tau_{i}\right) & =\int_{0}^{\infty} P\left(\tau_{i}<\hat{y} \mid \widehat{Y}=\widehat{y}\right) \mathrm{d} P(\widehat{Y} \leq \widehat{y}) \\
& =\int_{0}^{\infty} \xi_{i}(\hat{y}) \mathrm{d} F(\hat{y}), \quad 0 \leq y \leq 1,(i=1,2) .
\end{aligned}
$$

\section{System Analysis}

The states of this system $\Omega(t)$ at time $t$ as a following: 
(i) $\mathrm{S}_{0}$ : at any time $t$, unit $\mathrm{A}$ is active, unit $\mathrm{B}$ is active, and the repairman has the choice to either stay at the system or take a vacation.

(ii) $\mathrm{S}_{1}$ : at any time $t$, unit $\mathrm{A}$ is active, unit $\mathrm{B}$ is being repaired, the repairman is still in the system, and the system is working.

(iii) $\mathrm{S}_{2}$ : at any time $t$ unit $\mathrm{A}$ is being repaired, unit $\mathrm{B}$ is active, the repairman is still in the system, and the system is working.

(iv) $\mathrm{S}_{3}$ : at any time $t$, unit $\mathrm{A}$ is active, unit $\mathrm{B}$ is active, the repairman chooses to take a vacation, and the system is working.

(v) $\mathrm{S}_{4}$ : at any time $t$, unit $\mathrm{A}$ is still repaired from above $\mathrm{S}_{2}$, unit $\mathrm{B}$ is waiting for being repaired, and the system is down.

(vi) $\mathrm{S}_{5}$ : at any time $t$, unit $\mathrm{A}$ is active, unit $\mathrm{B}$ is waiting for repair, the repairman is on vacation, and the system is working.

(vii) $\mathrm{S}_{6}$ : at any time $t$, unit $\mathrm{A}$ is waiting for repair, unit $\mathrm{B}$ is active, the repairman is on vacation, and the system is working.

(viii) $\mathrm{S}_{7}$ : at any time $t$, unit $\mathrm{A}$ is still waiting for repair from $S_{6}$, unit $B$ is waiting for repair, the repairman is on vacation, and the system is down.

(ix) $\mathrm{S}_{8}$ : at any time $t$, unit $\mathrm{A}$ is waiting for being repaired, unit B is under repair, and the system is down.

(x) $\mathrm{S}_{9}$ : at any time $t$, unit $\mathrm{B}$ is still waiting for repair from $\mathrm{S}_{5}$, unit $\mathrm{A}$ is waiting for repair, the repairman is on vacation, and the system is down.

The state space is $E=\left\{\mathrm{S}_{0}, \mathrm{~S}_{1}, \mathrm{~S}_{2}, \mathrm{~S}_{3}, \mathrm{~S}_{4}, \mathrm{~S}_{5}, \mathrm{~S}_{6}, \mathrm{~S}_{7}, \mathrm{~S}_{8}, \mathrm{~S}_{9}\right\}$, where the working state is $U=\left\{\mathrm{S}_{0}, \mathrm{~S}_{1}, \mathrm{~S}_{2}, \mathrm{~S}_{3}, \mathrm{~S}_{5}, \mathrm{~S}_{6}\right\}$ and the down state is $D=\left\{S_{4}, S_{7}, S_{8}, S_{9}\right\}$ as shown in Figure 1 . According to the above assumptions, and $\{\Omega(t), t \geq 0\}$ is not a Markov process, we using the supplementary variables as a following:

(i) $X_{1}(t)$ is time to make a vacation decision. $\Omega(t)=\left\{\mathrm{S}_{0}\right\}$

(ii) $X_{2}(t)$ is the elapsed vacation time. $\Omega(t)=\left\{S_{5}, S_{6}, S_{7}, S_{9}\right\}$

(iii) $Y_{1}(t)$ is the elapsed repair time of unit A being repaired at time $t . \Omega(t)=\left\{\mathrm{S}_{2}, \mathrm{~S}_{4}\right\}$

(iv) $Y_{2}(t)$ is the elapsed repair time of unit $\mathrm{B}$ being repaired at time $t . \Omega(t)=\left\{\mathrm{S}_{1}, \mathrm{~S}_{8}\right\}$

State space is as follows: $\Omega^{*}=\{(0, u)$, $(1, y),(2, x), 3,(4, x),(5, z),(6, z),(7, z),(8, y),(9, z)\}$ where $u, z, x$, and $y$ are explanatory values of $X_{1}(t), X_{2}(t), Y_{1}(t)$, and $Y_{2}(t)$, respectively. Subject to

$$
\begin{aligned}
Q_{0}(t, u)=\rho\left(\Omega(t)=0, X_{1}(t) \leq u\right), & \\
Q_{i}(t, z)=\rho\left(\Omega(t)=i, X_{2}(t) \leq z\right), & (i=5,6,7,9), \\
Q_{i}(t, x)=\rho\left(\Omega(t)=i, Y_{1}(t) \leq x\right), & (i=2,4), \\
Q_{i}(t, y)=\rho\left(\Omega(t)=i, Y_{2}(t) \leq y\right), & (i=1,8),
\end{aligned}
$$

where $\rho(E)$ is the probability of event $E$.

$$
\begin{aligned}
\rho_{i}(t, w) & =\frac{\mathrm{d}}{\mathrm{dw}} Q_{i}(t, w), \quad(i=0,1,2,4,5,6,7,8,9), \\
\phi_{i}(t) & =\rho(s(t)=i), \quad(i=0,1,2,4,5,6,7,8,9) .
\end{aligned}
$$

From here, we can formulate the differential equations that represent this system by using the probability arguments and limiting transitions as follows:

$\rho_{0}(t+\Delta t, u+\Delta t)=\rho_{0}(t, u)\left(1-\left(\lambda_{1} r_{1}+\lambda_{2} r_{2}+\varphi(u)\right) \Delta t\right)+o \Delta t$.

When $\Delta t$ tend to zero, we get

$$
\left(\frac{\partial}{\partial t}+\frac{\partial}{\partial u}+\lambda_{1} r_{1}+\lambda_{2} r_{2}+\varphi(u)\right) \rho_{0}(t, u)=0 .
$$

In the same style, we get the following:

$$
\begin{aligned}
& \left(\frac{\partial}{\partial t}+\frac{\partial}{\partial y}+\lambda_{1} r_{1}+\mu_{2}(y)\right) \rho_{1}(t, y)=0, \\
& \left(\frac{\partial}{\partial t}+\frac{\partial}{\partial x}+\lambda_{2} r_{2}+\mu_{1}(x)\right) \rho_{2}(t, x)=0, \\
& \left(\frac{\mathrm{d}}{\mathrm{d} t}+\lambda_{1} r_{1}+\lambda_{2} r_{2}\right) \phi_{3}(t)=\int_{0}^{\infty} \rho_{0}(u, t) \varphi(u) \mathrm{du} \\
& \left(\frac{\partial}{\partial t}+\frac{\partial}{\partial x}+\mu_{1}(x)\right) \rho_{4}(t, x)=\lambda_{2} r_{2} \rho_{2}(t, x), \\
& \left(\frac{\partial}{\partial t}+\frac{\partial}{\partial z}+\lambda_{1} r_{1}+\alpha(z)\right) \rho_{5}(t, z)=0 \\
& \left(\frac{\partial}{\partial t}+\frac{\partial}{\partial z}+\lambda_{2} r_{2}+\alpha(z)\right) \rho_{6}(t, z)=0 \\
& \left(\frac{\partial}{\partial t}+\frac{\partial}{\partial z}+\alpha(z)\right) \rho_{7}(t, z)=\lambda_{2} r_{2} \rho_{6}(t, z) \\
& \left(\frac{\partial}{\partial t}+\frac{\partial}{\partial y}+\mu_{2}(y)\right) \rho_{8}(t, y)=\lambda_{1} r_{1} \rho_{1}(t, y), \\
& \left(\frac{\partial}{\partial t}+\frac{\partial}{\partial z}+\alpha(z)\right) \rho_{9}(t, z)=\lambda_{1} r_{1} \rho_{5}(t, z) .
\end{aligned}
$$

The boundary conditions are as follows:

$$
\rho_{0}(t, 0)=\int_{0}^{\infty} \rho_{1}(t, y) \mu_{2}(y) \mathrm{d} y+\int_{0}^{\infty} \rho_{2}(t, x) \mu_{1}(x) \mathrm{d} x+\varepsilon(t)
$$

$$
\begin{aligned}
\rho_{1}(t, 0)= & \int_{0}^{\infty} \lambda_{2} r_{2} \rho_{0}(t, u) \mathrm{d} u+\int_{0}^{\infty} \rho_{4}(t, x) \mu_{1}(x) \mathrm{d} x \\
& +\int_{0}^{\infty} \rho_{5}(t, z) \alpha(z) \mathrm{d} z
\end{aligned}
$$




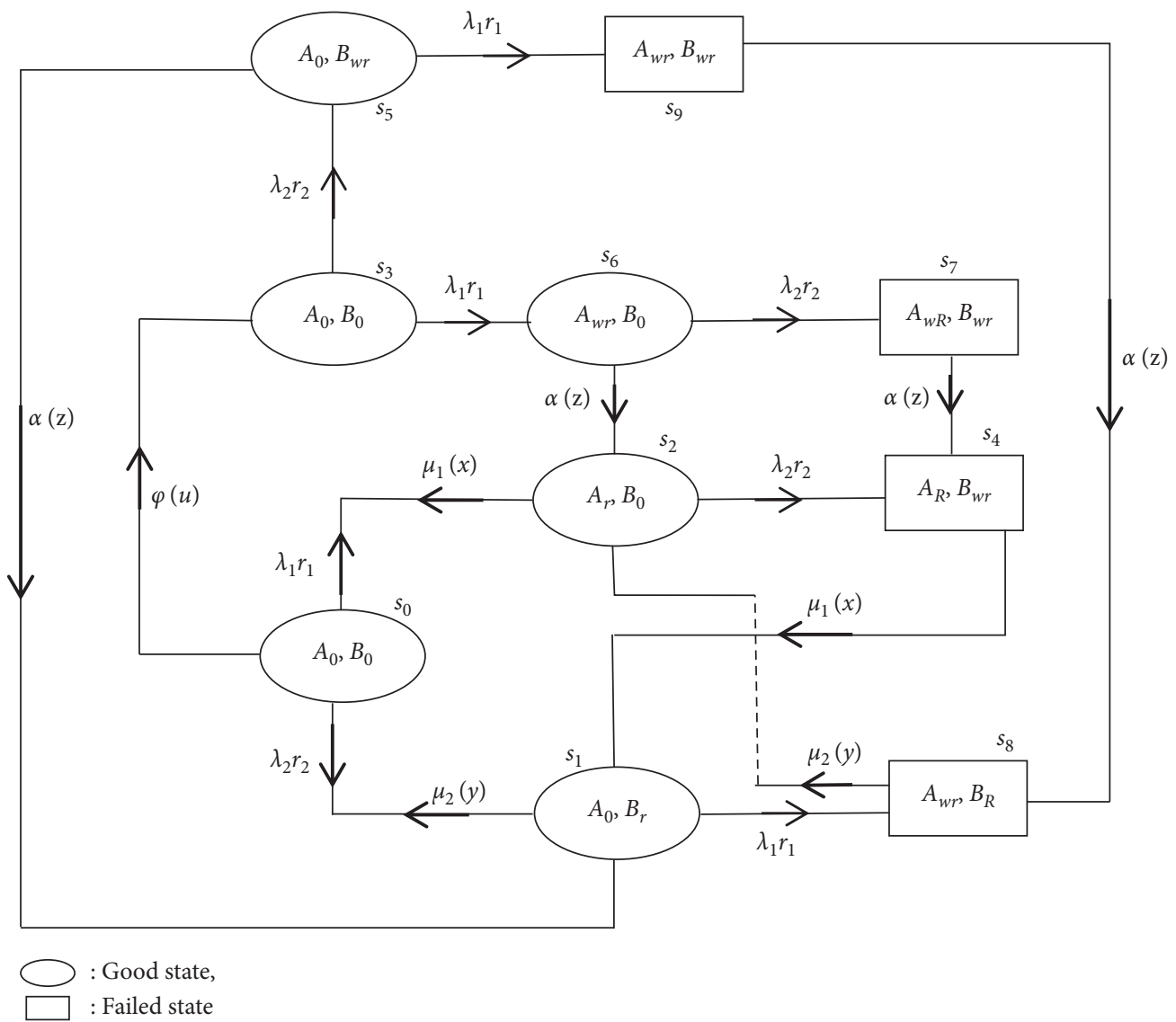

Figure 1: Transition diagram of the system.

$$
\begin{aligned}
\rho_{2}(t, 0)= & \int_{0}^{\infty} \lambda_{1} r_{1} \rho_{0}(t, u) \mathrm{d} u+\int_{0}^{\infty} \rho_{8}(t, y) \mu_{2}(y) \mathrm{d} y \\
& +\int_{0}^{\infty} \rho_{6}(t, z) \alpha(z) \mathrm{d} z, \\
\rho_{6}(t, 0)= & \lambda_{1} r_{1} \phi_{3}(t), \\
\rho_{6}(t, 0)= & \lambda_{1} r_{1} \phi_{3}(t), \\
\rho_{7}(t, 0)= & \rho_{9}(t, 0)=0, \\
\rho_{4}(t, 0)= & \int_{0}^{\infty} \rho_{7}(t, z) \alpha(z) \mathrm{d} z, \\
\rho_{8}(t, 0)= & \int_{0}^{\infty} \rho_{9}(t, z) \alpha(z) \mathrm{d} z,
\end{aligned}
$$

$$
\begin{aligned}
& \sum_{i=0}^{2} \int_{0}^{\infty} \rho_{i}(t, m) \mathrm{d} m+\phi_{3}(t) \\
& \quad+\sum_{i=4}^{9} \int_{0}^{\infty} \rho_{i}(t, m) \mathrm{d} m=1, \quad m=u, x, y, z .
\end{aligned}
$$

The initial conditions are as follows:

$$
\begin{aligned}
& \sum_{i=0}^{2} \int_{0}^{\infty} \rho_{i}(t, m) \mathrm{d} m+\phi_{3}(t) \\
& \quad+\sum_{i=4}^{9} \int_{0}^{\infty} \rho_{i}(t, m) \mathrm{d} m=1, \quad m=u, x, y, z .
\end{aligned}
$$

Laplace transform can be defined as follows: 


$$
k^{*}(s)=L\{k(x)\}=\int_{0}^{\infty} k(x) e^{-s x} \mathrm{~d} x, \quad s>0 .
$$

The ergodicity of the investigation process guarantees the presence of the following steady-probability: $\phi_{i}=\lim _{t \longrightarrow \infty} \phi_{i}(t), i=1, \ldots, 9 . \theta_{i}(m)=\lim _{t \longrightarrow \infty} \rho_{i}(t, m)$, $i=1,2,4,5,6,7,8,9$, which follows the following relations: $\phi_{i}=\int_{0}^{\infty} \theta_{i}(m) \mathrm{dm}, i=1,2,4,5,6,7,8,9$.

Taking the limit $t \longrightarrow \infty$ in the equations (6)-(23), the following equations are obtained:

$$
\begin{aligned}
& \left(\frac{\mathrm{d}}{\mathrm{d} u}+\lambda_{1} r_{1}+\lambda_{2} r_{2}+\varphi(u)\right) \theta_{0}(u)=0, \\
& \left(\frac{\mathrm{d}}{\mathrm{d} y}+\lambda_{1} r_{1}+\mu_{2}(y)\right) \theta_{1}(y)=0, \\
& \left(\frac{\partial}{\partial x}+\lambda_{2} r_{2}+\mu_{1}(x)\right) \theta_{2}(x)=0, \\
& \left(\lambda_{1} r_{1}+\lambda_{2} r_{2}\right) \phi_{3}=\int_{0}^{\infty} \theta_{0}(u) \varphi(u) \mathrm{d} u, \\
& \left(\frac{\mathrm{d}}{\mathrm{d} x}+\mu_{1}(x)\right) \theta_{4}(x)=\lambda_{2} r_{2} \theta_{2}(x), \\
& \left(\frac{\mathrm{d}}{\mathrm{d} z}+\lambda_{1} r_{1}+\alpha(z)\right) \theta_{5}(z)=0, \\
& \left(\frac{\partial}{\partial z}+\lambda_{2} r_{2}+\alpha(z)\right) \theta_{6}(z)=0, \\
& \left(\frac{d}{d z}+\alpha(z)\right) \theta_{7}(z)=\lambda_{2} r_{2} \theta_{6}(z), \\
& \left(\frac{\mathrm{d}}{\mathrm{d} y}+\mu_{2}(y)\right) \theta_{8}(y)=\lambda_{1} r_{1} \theta_{1}(y), \\
& \left(\frac{\mathrm{d}}{\mathrm{d} z}+\alpha(z)\right) \theta_{9}(z)=\lambda_{1} r_{1} \theta_{5}(z) \\
& \theta_{0}(0)=\int_{0}^{\infty} \theta_{1}(y) \mu_{2}(y) \mathrm{d} y+\int_{0}^{\infty} \theta_{2}(x) \mu_{1}(x) \mathrm{d} x, \\
& \theta_{1}(0)=\int_{0}^{\infty} \lambda_{2} r_{2} \theta_{0}(u) \mathrm{d} u+\int_{0}^{\infty} \theta_{4}(x) \mu_{1}(x) \mathrm{d} x+\int_{0}^{\infty} \theta_{5}(z) \alpha(z) \mathrm{d} z, \\
& \theta_{2}(0)=\int_{0}^{\infty} \lambda_{1} r_{1} \theta_{0}(u) \mathrm{d} u+\int_{0}^{\infty} \theta_{8}(y) \mu_{2}(y) \mathrm{d} y+\int_{0}^{\infty} \theta_{6}(z) \alpha(z) \mathrm{d} z, \\
& \theta_{5}(0)=\lambda_{2} r_{2} \phi_{3}, \\
& \theta_{6}(0)=\lambda_{1} r_{1} \phi_{3}
\end{aligned}
$$




$$
\begin{aligned}
\theta_{7}(0) & =\theta_{9}(0)=0, \\
\theta_{4}(0) & =\int_{0}^{\infty} \theta_{7}(z) \alpha(z) \mathrm{d} z, \\
\theta_{8}(0) & =\int_{0}^{\infty} \theta_{9}(z) \alpha(z) \mathrm{d} z, \\
\sum_{i=0}^{2} \int_{0}^{\infty} \theta_{i}(m) \mathrm{d} m+\phi_{3}+\sum_{i=4}^{9} \int_{0}^{\infty} \theta_{i}(m) \mathrm{d} m & =1, \quad m=u, x, y, z .
\end{aligned}
$$

Steady-state availability is as follows:

where

$\operatorname{Av}(\infty)=\phi_{0}+\phi_{1}+\phi_{2}+\phi_{3}+\phi_{5}+\phi_{6}$,

$$
\begin{aligned}
& \phi_{0}=C_{0} \bar{V}_{1}^{*}\left(\lambda_{1} r_{1}+\lambda_{2} r_{2}\right) \\
& \phi_{1}=\frac{C_{0}\left[a_{1}-\lambda_{1} r_{1}\left(\bar{V}_{1}^{*}\left(\lambda_{1} r_{1}+\lambda_{2} r_{2}\right)\right)\left(h_{1}^{*}\left(\lambda_{2} r_{2}\right)-1\right)+a_{2}\right] \bar{H}_{2}^{*}\left(\lambda_{1} r_{1}\right)}{h_{1}^{*}\left(\lambda_{2} r_{2}\right)\left(h_{2}^{*}\left(\lambda_{1} r_{1}\right)-1\right)-h_{2}^{*}\left(\lambda_{1} r_{1}\right)}, \\
& \phi_{2}=\frac{-\left[C_{0} \lambda_{1}^{2} r_{1}^{2} \bar{V}_{1}^{*}\left(\lambda_{1} r_{1}+\lambda_{2} r_{2}\right)+a_{3}+a_{4}\right] \bar{H}_{1}^{*}\left(\lambda_{2} r_{2}\right)}{\left(\lambda_{1} r_{1}+\lambda_{2} r_{2}\right)\left(h_{1}^{*}\left(\lambda_{2} r_{2}\right)\left(h_{2}^{*}\left(\lambda_{1} r_{1}\right)-1\right)-h_{2}^{*}\left(\lambda_{1} r_{1}\right)\right)}, \\
& \phi_{3}=\frac{C_{0} v_{1}^{*}\left(\lambda_{1} r_{1}+\lambda_{2} r_{2}\right)}{\lambda_{1} r_{1}+\lambda_{2} r_{2}} \\
& \phi_{5}=\frac{C_{0} \lambda_{2} r_{2} \bar{V}_{2}^{*}\left(\lambda_{1} r_{1}\right) v_{1}^{*}\left(\lambda_{1} r_{1}+\lambda_{2} r_{2}\right)}{\lambda_{1} r_{1}+\lambda_{2} r_{2}} \\
& \phi_{6}=\frac{C_{0} \lambda_{1} r_{1} \bar{V}_{2}^{*}\left(\lambda_{2} r_{2}\right) v_{1}^{*}\left(\lambda_{1} r_{1}+\lambda_{2} r_{2}\right)}{\lambda_{1} r_{1}+\lambda_{2} r_{2}}, \\
& C_{0}=\frac{\left[\alpha\left(\lambda_{1} r_{1}+\lambda_{2} r_{2}\right) \mu_{1} \mu_{2}\left(h_{1}^{*}\left(\lambda_{2} r_{2}\right)\left(h_{2}^{*}\left(\lambda_{1} r_{1}\right)-1\right)-h_{2}^{*}\left(\lambda_{1} r_{1}\right)\right)\right]}{a_{5}}, \\
& a_{1}=\frac{-\lambda_{2} r_{2}\left[\left(\lambda_{1} r_{1}+\lambda_{2} r_{2}\right) \bar{V}_{1}^{*}\left(\lambda_{1} r_{1}+\lambda_{2} r_{2}\right)+v_{1}^{*}\left(\lambda_{1} r_{1}+\lambda_{2} r_{2}\right)\left(1+h_{1}^{*}\left(\lambda_{2} r_{2}\right)\left(v_{2}^{*}\left(\lambda_{1} r_{1}\right)-1\right)\right)\right]}{\lambda_{1} r_{1}+\lambda_{2} r_{2}}, \\
& a_{2}=\frac{v_{1}^{*}\left(\lambda_{1} r_{1}+\lambda_{2} r_{2}\right)\left(1-h_{1}^{*}\left(\lambda_{2} r_{2}\right) v_{2}^{*}\left(\lambda_{2} r_{2}\right)\right)}{\lambda_{1} r_{1}+\lambda_{2} r_{2}} \\
& a_{3}=\lambda_{2} r_{2}\left[-\lambda_{2} r_{2} \bar{V}_{1}^{*}\left(\lambda_{1} r_{1}+\lambda_{2} r_{2}\right)\left(h_{2}^{*}\left(\lambda_{1} r_{1}\right)-1\right)+v_{1}^{*}\left(\lambda_{1} r_{1}+\lambda_{2} r_{2}\right)\left(1-h_{2}^{*}\left(\lambda_{1} r_{1}\right) v_{2}^{*}\left(\lambda_{1} r_{1}\right)\right)\right] \text {, } \\
& a_{4}=\lambda_{1} r_{1}\left[-\lambda_{2} r_{2} \bar{V}_{1}^{*}\left(\lambda_{1} r_{1}+\lambda_{2} r_{2}\right)\left(h_{2}^{*}\left(\lambda_{1} r_{1}\right)-2\right)+v_{1}^{*}\left(\lambda_{1} r_{1}+\lambda_{2} r_{2}\right)\left(1+h_{2}^{*}\left(\lambda_{1} r_{1}\right)\left(v_{2}^{*}\left(\lambda_{2} r_{2}\right)-1\right)\right)\right] \text {, } \\
& a_{5}=\alpha \lambda_{1}^{2} r_{1}^{2} \bar{V}_{1}^{*}\left(\lambda_{1} r_{1}+\lambda_{2} r_{2}\right)\left(-\mu_{2}+\mu_{1}\left(h_{1}^{*}\left(\lambda_{2} r_{2}\right)-1\right)\right)-\alpha \lambda_{2}^{2} r_{2}^{2} \bar{V}_{1}^{*}\left(\lambda_{1} r_{1}+\lambda_{2} r_{2}\right)\left(\mu_{1}-\mu_{2}\left(h_{2}^{*}\left(\lambda_{1} r_{1}\right)-1\right)\right) \text {, } \\
& +\alpha \mu_{2} \mu_{1}\left(h_{1}^{*}\left(\lambda_{2} r_{2}\right)\left(h_{2}^{*}\left(\lambda_{1} r_{1}\right)-1\right)-h_{2}^{*}\left(\lambda_{1} r_{1}\right)\right) v_{1}^{*}\left(\lambda_{1} r_{1}+\lambda_{2} r_{2}\right)+\lambda_{2} r_{2}\left(\mu _ { 1 } \left(\mu _ { 2 } \left(-h_{1}^{*}\left(\lambda_{2} r_{2}\right)+\left(h_{1}^{*}\left(\lambda_{2} r_{2}\right)-1\right),\right.\right.\right. \\
& \left.h_{2}^{*}\left(\lambda_{1} r_{1}\right)\right)\left(\alpha \bar{V}_{1}^{*}\left(\lambda_{1} r_{1}+\lambda_{2} r_{2}\right)+v_{1}^{*}\left(\lambda_{1} r_{1}+\lambda_{2} r_{2}\right)\right)-\alpha v_{1}^{*}\left(\lambda_{1} r_{1}+\lambda_{2} r_{2}\right)\left(1+\left(h_{1}^{*}\left(\lambda_{2} r_{2}\right)-1\right) h_{2}^{*}\left(\lambda_{1} r_{1}\right)\right. \text {, } \\
& \left.\left.\left.\left(v_{2}^{*}\left(\lambda_{1} r_{1}\right)-1\right)\right)\right)+\alpha \mu_{2} v_{1}^{*}\left(\lambda_{1} r_{1}+\lambda_{2} r_{2}\right)\left(v_{2}^{*}\left(\lambda_{1} r_{1}\right) h_{2}^{*}\left(\lambda_{1} r_{1}\right)-1\right)\right)+\lambda_{1} r_{1}\left(\alpha \lambda_{2} r_{2} \bar{V}_{1}^{*}\left(\lambda_{1} r_{1}+\lambda_{2} r_{2}\right),\right.
\end{aligned}
$$




$$
\begin{aligned}
& \left(\mu_{1}\left(h_{1}^{*}\left(\lambda_{2} r_{2}\right)-2\right)+\mu_{2}\left(h_{2}^{*}\left(\lambda_{1} r_{1}\right)-2\right)\right)-\alpha \mu_{2} v_{1}^{*}\left(\lambda_{1} r_{1}+\lambda_{2} r_{2}\right)\left(1+h_{1}^{*}\left(\lambda_{2} r_{2}\right)\left(h_{2}^{*}\left(\lambda_{1} r_{1}\right)-1\right)\left(v_{2}^{*}\left(\lambda_{2} r_{2}-1\right)\right)\right), \\
& \quad+\mu_{1}\left(\mu_{2}\left(\left(h_{1}^{*}\left(\lambda_{2} r_{2}\right)-1\right) h_{2}^{*}\left(\lambda_{1} r_{1}\right)-h_{1}^{*}\left(\lambda_{2} r_{2}\right)\right)\left(v_{1}^{*}\left(\lambda_{1} r_{1}+\lambda_{2} r_{2}\right)+\alpha \bar{V}_{1}^{*}\left(\lambda_{1} r_{1}+\lambda_{2} r_{2}\right)\right),\right. \\
& \left.\left.\left.\quad+\alpha v_{1}^{*}\left(\lambda_{1} r_{1}+\lambda_{2} r_{2}\right)\left(v_{2}^{*}\left(\lambda_{2} r_{2}\right) h_{1}^{*}\left(\lambda_{2} r_{2}\right)-1\right)\right)\right)\right) .
\end{aligned}
$$

\section{Mean Time to the First Failure (MTTFF)}

In this section, we deduce the mean time to the first failure (MTTFF) of the system. We assumed that $t$ be the time to the first failure of the system; therefore, the reliability function of this system is calculated as follows: $R(t)=P(\tau>t)$. To obtain the reliability function, we consider the failure states $\{4,7,8,9\}$ of the system are absorbing states.

Let

$$
\begin{aligned}
L_{0}(t, u) & =\frac{\mathrm{d}}{\mathrm{d} u} \rho\left[\widetilde{S}(t)=0, \widetilde{X}_{1}(t) \leq u\right], \\
L_{i}(t, u) & =\frac{\mathrm{d}}{\mathrm{d} z} \rho\left[\widetilde{S}(t)=i, \widetilde{X}_{2}(t) \leq z\right], \quad i=5,6, \\
L_{1}(t, y) & =\frac{\mathrm{d}}{\mathrm{d} y} \rho\left[\widetilde{S}(t)=1, \widetilde{Y}_{2}(t) \leq y\right], \\
L_{3}(t) & =\rho[\widetilde{S}(t)=3], \\
L_{2}(t, x) & =\frac{\mathrm{d}}{\mathrm{d} x} \rho\left[\widetilde{S}(t)=2, \widetilde{Y}_{1}(t) \leq x\right] .
\end{aligned}
$$

In the same manner as previously mentioned in Section 3 , we conclude the reliability function as follows:

$$
\begin{aligned}
\left(\frac{\partial}{\partial t}+\frac{\partial}{\partial u}+\lambda_{1} r_{1}+\lambda_{2} r_{2}+\varphi(u)\right) L_{0}(t, u) & =0, \\
\left(\frac{\partial}{\partial t}+\frac{\partial}{\partial y}+\lambda_{1} r_{1}+\mu_{2}(y)\right) L_{1}(t, y) & =0, \\
\left(\frac{\partial}{\partial t}+\frac{\partial}{\partial x}+\lambda_{2} r_{2}+\mu_{1}(x)\right) L_{2}(t, x) & =0,
\end{aligned}
$$

$$
\begin{gathered}
\left(\frac{\mathrm{d}}{\mathrm{d} t}+\lambda_{1} r_{1}+\lambda_{2} r_{2}\right) L_{3}(t)=\int_{0}^{\infty} L_{0}(u, t) \varphi(u) \mathrm{du}, \\
\left(\frac{\partial}{\partial t}+\frac{\partial}{\partial z}+\lambda_{1} r_{1}+\alpha(z)\right) L_{5}(t, z)=0, \\
\left(\frac{\partial}{\partial t}+\frac{\partial}{\partial z}+\lambda_{2} r_{2}+\alpha(z)\right) L_{6}(t, z)=0 .
\end{gathered}
$$

The boundary conditions are as follows:

$$
\begin{aligned}
& L_{0}(t, 0)=\int_{0}^{\infty} L_{1}(t, y) \mu_{2}(y) \mathrm{d} y+\int_{0}^{\infty} L_{2}(t, x) \mu_{1}(x) \mathrm{d} x+\varepsilon(t) \\
& L_{1}(t, 0)=\int_{0}^{\infty} \lambda_{2} r_{2} L_{0}(t, u) \mathrm{d} u+\int_{0}^{\infty} L_{5}(t, z) \alpha(z) \mathrm{d} z \\
& L_{2}(t, 0)=\int_{0}^{\infty} \lambda_{1} r_{1} L_{0}(t, u) \mathrm{d} u+\int_{0}^{\infty} L_{6}(t, z) \alpha(z) \mathrm{d} z \\
& L_{5}(t, 0)=\lambda_{2} r_{2} L_{3}(t) \\
& L_{6}(t, 0)=\lambda_{1} r_{1} L_{3}(t) .
\end{aligned}
$$

The initial conditions are as follows:

$$
R_{0}(0, u)=\varepsilon(u)= \begin{cases}1, & u=0, \\ 0, & u \neq 0 .\end{cases}
$$

Taking the Laplace transform of the equations (30)-(40), as well as initial conditions, we have

$$
\begin{aligned}
\left(\frac{\mathrm{d}}{\mathrm{d} u}+s+\lambda_{1} r_{1}+\lambda_{2} r_{2}+\varphi(u)\right) L_{0}^{*}(s, u) & =0, \\
\left(\frac{\mathrm{d}}{\mathrm{d} y}+s+\lambda_{1} r_{1}+\mu_{2}(y)\right) L_{1}^{*}(s, y) & =0, \\
\left(\frac{\partial}{\partial x}+s+\lambda_{2} r_{2}+\mu_{1}(x)\right) L_{2}^{*}(s, x) & =0, \\
\left(s+\lambda_{1} r_{1}+\lambda_{2} r_{2}\right) L_{3}^{*}(s) & =\int_{0}^{\infty} L_{0}^{*}(u, s) \varphi(u) \mathrm{d} u,
\end{aligned}
$$




$$
\begin{aligned}
\left(\frac{\mathrm{d}}{\mathrm{d} z}+s+\lambda_{1} r_{1}+\alpha(z)\right) L_{5}^{*}(s, z) & =0 \\
\left(\frac{\mathrm{d}}{\mathrm{d} z}+s+\lambda_{2} r_{2}+\alpha(z)\right) L_{6}^{*}(s, z) & =0 \\
L_{0}^{*}(s, 0) & =\int_{0}^{\infty} L_{1}^{*}(s, y) \mu_{2}(y) \mathrm{d} y+\int_{0}^{\infty} L_{2}^{*}(s, x) \mu_{1}(x) \mathrm{d} x+\varepsilon(t), \\
L_{1}^{*}(s, 0) & =\int_{0}^{\infty} \lambda_{2} r_{2} L_{0}^{*}(s, u) \mathrm{d} u+\int_{0}^{\infty} L_{5}^{*}(s, z) \alpha(z) \mathrm{d} z, \\
L_{2}^{*}(s, 0) & =\int_{0}^{\infty} \lambda_{1} r_{1} L_{0}^{*}(s, u) \mathrm{d} u, \\
L_{5}^{*}(s, 0) & =\lambda_{2} r_{2} L_{3}^{*}(s), \\
L_{6}^{*}(s, 0) & =\lambda_{1} r_{1} L_{3}^{*}(s) .
\end{aligned}
$$

From the previous equations, we defined the reliability function as follows:

$$
\begin{aligned}
R^{*}(s)= & \int_{0}^{\infty} L_{0}^{*}(s, u) \mathrm{d} u+\int_{0}^{\infty} L_{1}^{*}(s, y) \mathrm{d} y \\
& +\int_{0}^{\infty} L_{2}^{*}(s, x) \mathrm{d} x+\sum_{i=5}^{6} \int_{0}^{\infty} L_{i}^{*}(s, z) \mathrm{d} z+L_{3}^{*}(s),
\end{aligned}
$$

and the mean time to the first failure of the system (MTTFF) is given by

$$
\operatorname{MTTFF}=\lim _{s \longrightarrow 0} R^{*}(s),
$$

$$
\begin{aligned}
L_{0}^{*}(s)= & \int_{0}^{\infty} L_{0}^{*}(s, u) \mathrm{d} u=L_{0}^{*}(s, 0) \bar{V}_{1}^{*}\left(s+r_{1} \lambda_{1}+r_{2} \lambda_{2}\right), \\
L_{1}^{*}(s)= & \int_{0}^{\infty} L_{1}^{*}(s, y) \mathrm{d} y=L_{1}^{*}(s, 0) \bar{H}_{2}^{*}\left(s+r_{1} \lambda_{1}\right), \\
L_{2}^{*}(s)= & \int_{0}^{\infty} L_{2}^{*}(s, x) \mathrm{d} x=L_{2}^{*}(s, 0) \bar{H}_{1}^{*}\left(s+r_{2} \lambda_{2}\right), \\
L_{3}^{*}(s)= & \frac{L_{0}^{*}(s, 0) v_{1}^{*}\left(s+r_{1} \lambda_{1}+r_{2} \lambda_{2}\right)}{s+r_{1} \lambda_{1}+r_{2} \lambda_{2}}, \\
L_{5}^{*}(s)= & \int_{0}^{\infty} L_{5}^{*}(s, z) \mathrm{d} z=L_{5}^{*}(s, 0) \bar{V}_{2}^{*}\left(s+r_{1} \lambda_{1}\right), \\
L_{6}^{*}(s)= & \int_{0}^{\infty} L_{6}^{*}(s, z) \mathrm{d} z=L_{6}^{*}(s, 0) \bar{V}_{2}\left(s+r_{2} \lambda_{2}\right), \\
& \left.\left.+r_{2} \lambda_{2} h_{2}^{*}\left(s+r_{1} \lambda_{1}\right)\right) \bar{V}_{1}^{*}\left(s+r_{1} \lambda_{1}+r_{2} \lambda_{2}\right)\right\} \\
L_{0}^{*}(s, 0)= & \frac{\left\{\varepsilon^{*}(s)\left(s+r_{1} \lambda_{1}+r_{2} \lambda_{2}\right)\right\}}{\left\{-s+r_{2} \lambda_{2}\left(-1+h_{2}^{*}\left(s+r_{1} \lambda_{1}\right) v_{1}^{*}\left(s+r_{1} \lambda_{1}+r_{2} \lambda_{2}\right) v_{2}^{*}\left(s+r_{1} \lambda_{1}\right)\right),\right.} \\
& +r_{1}\left(s+h_{1}^{*}\left(s+r_{2} \lambda_{2}\right) v_{1}^{*}\left(s+r_{1} \lambda_{1}+r_{2} \lambda_{2}\right) v_{2}^{*}\left(s+r_{2} \lambda_{2}\right)\right)+\left(s+r_{1} \lambda_{1}+r_{2} \lambda_{2}\right)\left(r_{1} \lambda_{1} h_{1}^{*}\left(s+r_{2} \lambda_{2}\right),\right.
\end{aligned}
$$




$$
\begin{aligned}
& L_{1}^{*}(s, 0)=r_{2} \lambda_{2} L_{0}^{*}(s, 0)\left\{\bar{V}_{1}^{*}\left(s+r_{1} \lambda_{1}+r_{2} \lambda_{2}\right)+\left\{\frac{v_{1}^{*}\left(s+r_{1} \lambda_{1}+r_{2} \lambda_{2}\right) v_{2}^{*}\left(s+r_{2} \lambda_{2}\right)}{\left(s+r_{1} \lambda_{1}+r_{2} \lambda_{2}\right)}\right\}\right\}, \\
& L_{2}^{*}(s, 0)=r_{1} \lambda_{1} L_{0}^{*}(s, 0)\left\{\bar{V}_{1}^{*}\left(s+r_{1} \lambda_{1}+r_{2} \lambda_{2}\right)+\left\{\frac{v_{1}^{*}\left(s+r_{1} \lambda_{1}+r_{2} \lambda_{2}\right) v_{2}^{*}\left(s+r_{2} \lambda_{2}\right)}{\left(s+r_{1} \lambda_{1}+r_{2} \lambda_{2}\right)}\right\}\right\}, \\
& L_{5}^{*}(s, 0)=\frac{\left\{r_{2} \lambda_{2} L_{0}^{*}(s, 0) v_{1}^{*}\left(s+r_{1} \lambda_{1}+r_{2} \lambda_{2}\right)\right\}}{\left(s+r_{1} \lambda_{1}+r_{2} \lambda_{2}\right)} \\
& L_{6}^{*}(s, 0)=\frac{\left\{r_{1} \lambda_{1} L_{0}^{*}(s, 0) v_{1}^{*}\left(s+r_{1} \lambda_{1}+r_{2} \lambda_{2}\right)\right\}}{\left(s+r_{1} \lambda_{1}+r_{2} \lambda_{2}\right)}
\end{aligned}
$$

\section{Numerical Analysis and Discussion}

In this section, the usefulness of the proposed system is demonstrated by examining the effects of the number of shocks and other parameters on the system using the following numerical figures, taking into account that

$$
\begin{aligned}
& V_{1}(t)= \begin{cases}1-e^{-\varphi t}, & t>0, \\
0, & t \leq 0,\end{cases} \\
& V_{2}(t)= \begin{cases}1-e^{-\alpha t}, & t>0, \\
0, & t \leq 0,\end{cases} \\
& H_{i}(t)=\left\{\begin{array}{ll}
1-e^{-\mu_{i} t}, & t>0, \\
0, & t \leq 0,
\end{array} \quad \forall i=1,2 .\right.
\end{aligned}
$$

The study of the behavior of steady-state availability and mean time to system failure for the number of shocks can be clearly seen in Figures $2-9$, respectively. Figures $2-5$ show the effects of both $r_{1}$ and $r_{2}$ on steady-state availability versus the effects of various system parameters. Likewise, Figures 6-5 discuss the same effects of both $r_{1}$ and $r_{2}$ on mean time to system failure against various system parameters. These results are summarized as follows.

In Figures 2 and 6, we can clearly observe the effects of the different cases of $r_{1}$ and $r_{2}$ on the steady-state availability and mean time to system failure against $\mu_{1} \in[0,1]$ at $\left(\varphi=0.2, \alpha=0.1, \lambda_{1}=0.4, \lambda_{2}=0.5, \mu_{2}=0.5\right)$. The steadystate availability and the mean time to system failure are increased when $r_{1}=r_{2}=0.2$, and the curves start to decrease gradually when $r_{1}=0.2<r_{2}=0.4$ and $r_{1}=0.4>r_{2}=$ 0.2 , respectively.

Figures 3 and 7 show the effect of $r_{1}$ and $r_{2}$ on steadystate availability and mean time to system failure versus $\mu_{2} \in[0,1]$ at $\left(\varphi=0.2, \alpha=0.2, \lambda_{1}=0.4, \lambda_{2}=0.5, \mu_{1}=0.6\right)$. The steady-state availability and mean time to system failure increase in the case of $r_{1}=r_{2}=0.2$, while they decrease regularly when $r_{1}=0.2<r_{2}=0.4$ and $r_{1}=0.4>r_{2}=0.2$.

In Figures 4 and 8 , we can discuss the effects of parameters $r_{1}$ and $r_{2}$ on steady-state availability and mean time to system failure against parameter $\alpha \in[0,1]$ when $\left(\varphi=0.2, \lambda_{1}=0.4, \lambda_{2}=0.5, \mu_{1}=0.6, \mu_{2}=0.5\right)$. The steadystate availability and mean time to system failure decrease in the cases $r_{1}=0.2<r_{2}=0.4$ and $r_{1}=0.4>r_{2}=0.2$, but they increase when $r_{1}=r_{2}=0.2$.

The effects of parameters $r_{1}$ and $r_{2}$ on steady-state availability and mean time to system failure against parameter $\varphi \in[0,1]$ at $\left(\alpha=0.2, \lambda_{1}=0.4, \lambda_{2}=0.5, \mu_{1}=\right.$ $\left.0.6, \mu_{2}=0.5\right)$ can be seen in Figures 5 and 9. From these curves, it can see that the steady-state availability and mean time to system failure increase when $r_{1}=r_{2}=0.2$, while they gradually decrease when $r_{1}=0.2<r_{2}=0.4$ and $r_{1}=0.4>r_{2}=0.2$.

From Figures 2-9, we can come to the following conclusion:

(1) In the steady-state availability, $\operatorname{Av}(\infty)$ increase when $r_{1}=r_{2}=0.2$

(2) In the mean time to system failure, MTTFF increase when $r_{1}=r_{2}=0.2$

\section{Sensitivity and Relative Sensitivity Analysis}

In this section, we perform sensitivity and relative sensitivity analysis of mean time to system failure (MTTFF) and steadystate availability, $\operatorname{Av}(\infty)$ along with changes in certain values of the system parameters $\varphi, \lambda_{1}, \lambda_{2}, \mu_{1}, \mu_{2}$, and $\alpha$.

6.1. Sensitivity and Relative Sensitivity Analysis for MTTFF. We defined the sensitivity analysis for MTTFF via the differentiation of (44) with respect to $\kappa$.

$$
\eta_{\kappa}=\frac{\partial \mathrm{MTTFF}}{\partial \kappa} \text {. }
$$

Moreover, relative sensitivity analysis for MTTFF defined as

$$
\theta_{\kappa}=\eta_{\kappa} \frac{\kappa}{\mathrm{MTTFF}},
$$

where $\kappa=\varphi, \lambda_{1}, \lambda_{2}, \mu_{1}, \mu_{2}, \alpha$.

6.2. Sensitivity and Relative Sensitivity Analysis for $(A v(\infty))$. We defined sensitivity analysis for $\operatorname{Av}(\infty)$ via differentiating (27) with respect to $\kappa$.

$$
\sigma_{\kappa}=\frac{\partial \operatorname{Av}(\infty)}{\partial \kappa}
$$

In addition, relative sensitivity analysis of $\operatorname{Av}(\infty)$ can be written in the form

$$
\prod_{\kappa}=\sigma_{k} \frac{k}{\operatorname{Av}(\infty)}
$$




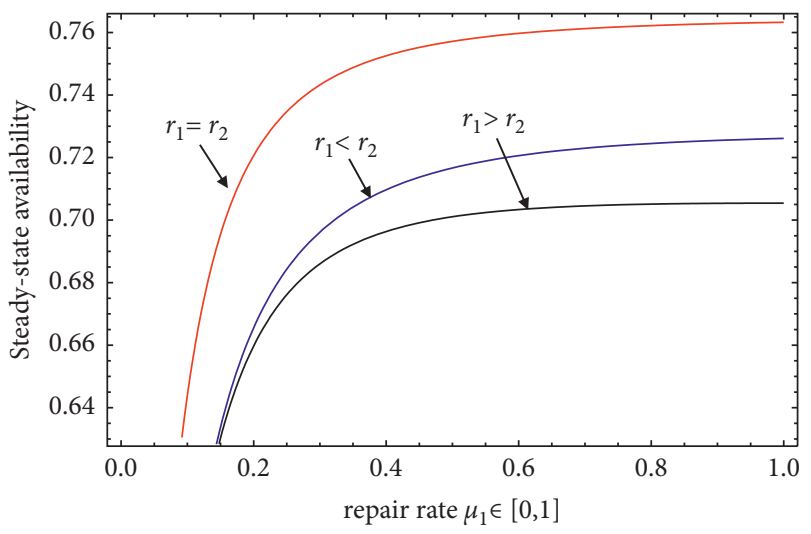

Figure 2: Steady-state availability versus rate $\mu_{1}$ when $\varphi=0.2, \alpha=0.1, \lambda_{1}=0.4, \lambda_{2}=0.5$, and $\mu_{2}=0.5$.

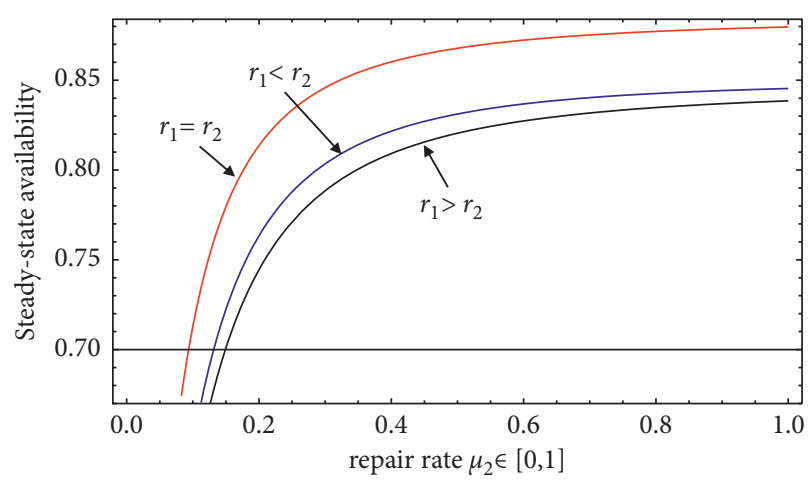

Figure 3: Steady-state availability versus rate $\mu_{2}$ when $\varphi=0.2, \alpha=0.1, \lambda_{1}=0.4, \lambda_{2}=0.5$, and $\mu_{1}=0.6$.

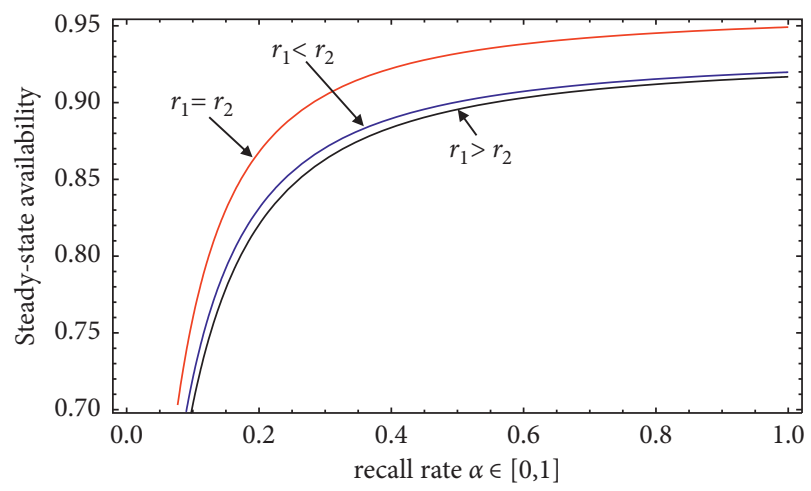

Figure 4: Steady-state availability versus rate $\alpha$ when $\varphi=0.2, \lambda_{1}=0.4, \lambda_{2}=0.5, \mu_{1}=0.6$, and $\mu_{2}=0.5$.

The results of the sensitivity analysis to the MTTFF affected by each system parameter are shown in Tables 1-3. From these tables, the order of the sensitivity analysis influenced by each parameter can be derived as follows:

(1) In the case $r_{1}=r_{2}$, we get $\lambda_{1}>\lambda_{2}>\varphi>\alpha>\mu_{2}>\mu_{1}$

(2) In the case $r_{1}>r_{2}$, we get $\lambda_{2}>\lambda_{1}>\varphi>\alpha>\mu_{2}>\mu_{1}$

(3) In the case $r_{1}<r_{2}$, we get $\lambda_{1}>\lambda_{2}>\varphi>\alpha>\mu_{2}>\mu_{1}$

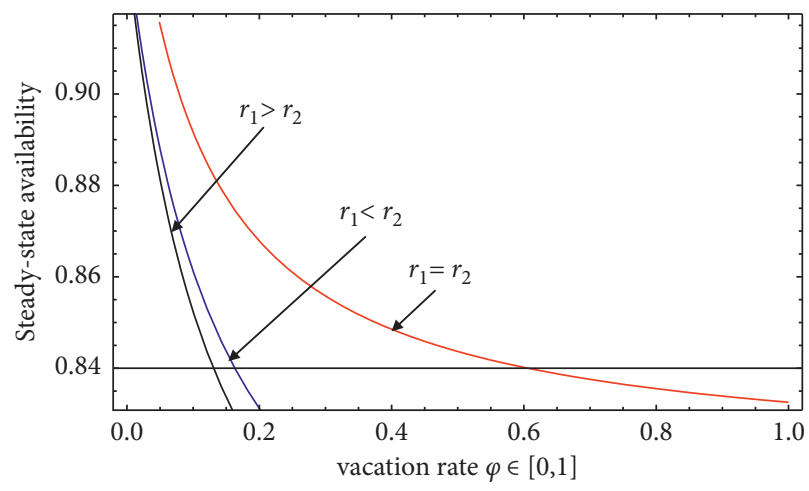

Figure 5: Steady-state availability versus rate $\varphi$ when $\alpha=0.2, \lambda_{1}=0.4, \lambda_{2}=0.5, \mu_{1}=0.6$, and $\mu_{2}=0.5$.

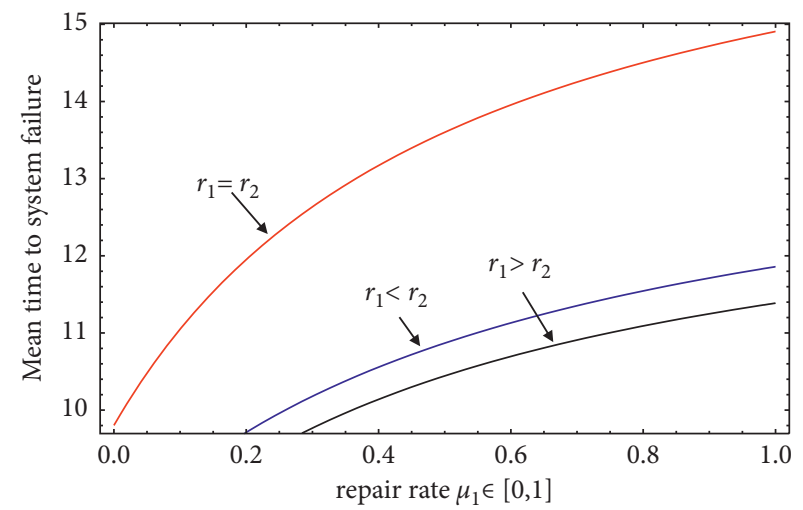

Figure 6: Mean time to system failure versus rate $\mu_{1}$ when $\varphi=0.3, \alpha=0.5, \lambda_{1}=0.4, \lambda_{2}=0.5$, and $\mu_{2}=0.5$.

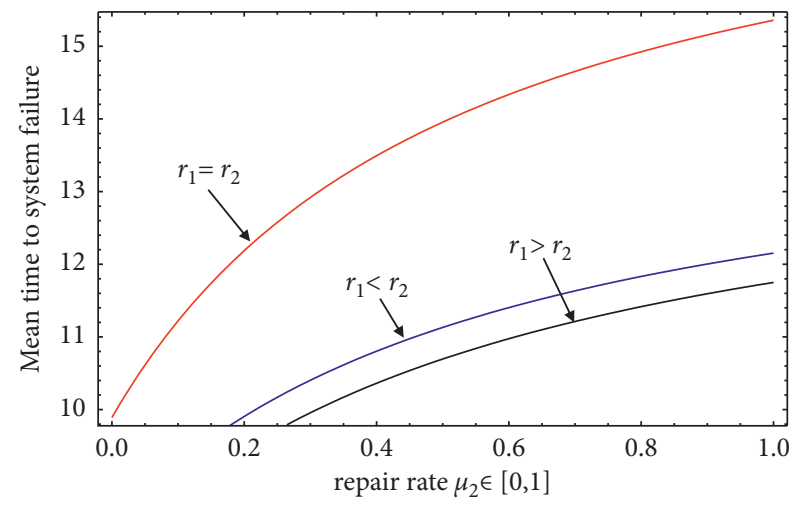

Figure 7: Mean time to system failure versus rate $\mu_{2}$ when $\varphi=0.3, \alpha=0.5, \lambda_{1}=0.4, \lambda_{2}=0.5$, and $\mu_{1}=0.6$.

Moreover, the signs of the sensitivity to MTTFF affected by the parameters $\lambda_{1}, \lambda_{2}$ and $\varphi$ are negative, which means that increasing the parameter worsens the sensitivity to MTTFF; the signs of the sensitivity to MTTFF affected by the parameters $\mu_{1}, \mu_{2}$ and $\alpha$ are positive, which means that increasing the parameter improves the sensitivity to MTTFF.

The relative sensitivity is completed. The results of relative sensitivity for MTTFF can be found in Tables 4-6. 


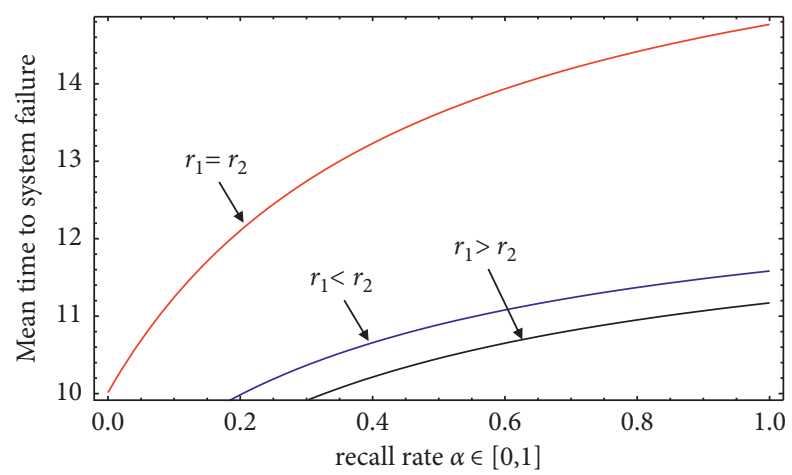

Figure 8: Mean time to system failure versus rate $\alpha$ when $\varphi=0.4, \lambda_{1}=0.4, \lambda_{2}=0.5, \mu_{1}=0.6$, and $\mu_{2}=0.5$.

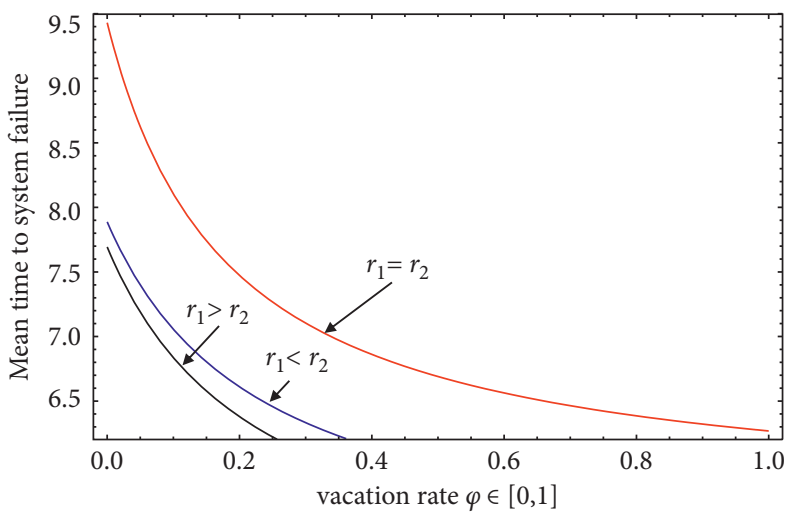

Figure 9: Mean time to system failure versus rate $\varphi$ when $\alpha=0.01, \lambda_{1}=0.4, \lambda_{2}=0.5, \mu_{1}=0.6$, and $\mu_{2}=0.5$.

TABLE 1: Sensitivity of MTTFF when $r_{1}=r_{2}$

\begin{tabular}{cccccccccccc}
\hline$\lambda_{1}$ & $\eta_{\lambda_{1}}$ & $\lambda_{2}$ & $\eta_{\lambda_{2}}$ & $\mu_{1}$ & $\eta_{\mu_{1}}$ & $\mu_{2}$ & $\eta_{\mu_{2}}$ & $\alpha$ & $\eta_{\alpha}$ & $\varphi$ & $\eta_{\varphi}$ \\
\hline 0.1 & -685.96 & 0.1 & -654.43 & 0.1 & 13.2637 & 0.1 & 13.692 & 0.1 & 31.2202 & 0.1 & -46.418 \\
0.2 & -169.461 & 0.2 & -161.91 & 0.2 & 7.55393 & 0.2 & 8.00739 & 0.2 & 22.0348 & 0.2 & -17.651 \\
0.3 & -74.1623 & 0.3 & -70.987 & 0.3 & 4.87081 & 0.3 & 5.24772 & 0.3 & 16.379 & 0.3 & -9.2242 \\
0.4 & -41.0233 & 0.4 & -39.340 & 0.4 & 3.39947 & 0.4 & 3.70291 & 0.4 & 12.6511 & 0.4 & -5.6555 \\
0.5 & -25.8214 & 0.5 & -24.805 & 0.5 & 2.50653 & 0.5 & 2.75193 & 0.5 & 10.065 & 0.5 & -3.8186 \\
\hline
\end{tabular}

TABLE 2: Sensitivity of MTTFF when $r_{1}>r_{2}$.

\begin{tabular}{cccccccccccc}
\hline$\lambda_{1}$ & $\eta_{\lambda_{1}}$ & $\lambda_{2}$ & $\eta_{\lambda_{2}}$ & $\mu_{1}$ & $\eta_{\mu_{1}}$ & $\mu_{2}$ & $\eta_{\mu_{2}}$ & $\alpha$ & $\eta_{\alpha}$ & $\varphi$ & $\eta_{\varphi}$ \\
\hline 0.1 & -338.921 & 0.1 & -587.30 & 0.1 & 7.59107 & 0.1 & 7.76191 & 0.1 & 13.8699 & 0.1 & -24.243 \\
0.2 & -82.0465 & 0.2 & -146.14 & 0.2 & 4.67982 & 0.2 & 5.31203 & 0.2 & 9.67936 & 0.2 & -10.931 \\
0.3 & -35.3059 & 0.3 & -64.545 & 0.3 & 3.17101 & 0.3 & 3.86245 & 0.3 & 7.1666 & 0.3 & -6.1934 \\
0.4 & -19.3399 & 0.4 & -36.049 & 0.4 & 2.28962 & 0.4 & 2.93438 & 0.4 & 5.5277 & 0.4 & -3.9811 \\
0.5 & -12.1429 & 0.5 & -22.902 & 0.5 & 1.73047 & 0.5 & 2.30465 & 0.5 & 4.39606 & 0.5 & -2.7727 \\
\hline
\end{tabular}

TABLE 3: Sensitivity of MTTFF when $r_{1}<r_{2}$.

\begin{tabular}{cccccccccccc}
\hline$\lambda_{1}$ & $\eta_{\lambda_{1}}$ & $\lambda_{2}$ & $\eta_{\lambda_{2}}$ & $\mu_{1}$ & $\eta_{\mu_{1}}$ & $\mu_{2}$ & $\eta_{\mu_{2}}$ & $\alpha$ & $\eta_{\alpha}$ & $\varphi$ & $\eta_{\varphi}$ \\
\hline 0.1 & -603.243 & 0.1 & -323.82 & 0.1 & 7.56729 & 0.1 & 7.94314 & 0.1 & 14.1419 & 0.1 & -24.669 \\
0.2 & -149.952 & 0.2 & -78.680 & 0.2 & 5.00491 & 0.2 & 4.96722 & 0.2 & 9.93321 & 0.2 & -11.018 \\
0.3 & -66.1384 & 0.3 & -33.969 & 0.3 & 3.55372 & 0.3 & 3.39711 & 0.3 & 7.36809 & 0.3 & -6.2125 \\
0.4 & -36.886 & 0.4 & -18.651 & 0.4 & 2.65307 & 0.4 & 2.46889 & 0.4 & 5.68493 & 0.4 & -3.9818 \\
0.5 & -23.4 & 0.5 & -11.727 & 0.5 & 2.05599 & 0.5 & 1.87499 & 0.5 & 4.51999 & 0.5 & -2.7679 \\
\hline
\end{tabular}


TABLE 4: Relative sensitivity of MTTFF when $r_{1}=r_{2}$.

\begin{tabular}{cccccccccccc}
\hline$\lambda_{1}$ & $\theta_{\lambda_{1}}$ & $\lambda_{2}$ & $\theta_{\lambda_{2}}$ & $\mu_{1}$ & $\theta_{\mu_{1}}$ & $\mu_{2}$ & $\theta_{\mu_{2}}$ & $\alpha$ & $\theta_{\alpha}$ & $\varphi$ & $\theta_{\varphi}$ \\
\hline 0.1 & -0.8978 & 0.1 & -0.9107 & 0.1 & 0.07543 & 0.1 & 0.07756 & 0.1 & 0.1553 & 0.1 & -0.1914 \\
0.2 & -0.8023 & 0.2 & -0.8249 & 0.2 & 0.08129 & 0.2 & 0.08564 & 0.2 & 0.1939 & 0.2 & -0.1651 \\
0.3 & -0.7172 & 0.3 & -0.7466 & 0.3 & 0.07614 & 0.3 & 0.0814 & 0.3 & 0.1995 & 0.3 & -0.1376 \\
0.4 & -0.6435 & 0.4 & -0.6773 & 0.4 & 0.06939 & 0.4 & 0.07485 & 0.4 & 0.1941 & 0.4 & -0.1167 \\
0.5 & -0.5804 & 0.5 & -0.6168 & 0.5 & 0.06301 & 0.5 & 0.06843 & 0.5 & 0.1851 & 0.5 & -0.1009 \\
\hline
\end{tabular}

TABLE 5: Relative sensitivity of MTTFF when $r_{1}>r_{2}$.

\begin{tabular}{cccccccccccc}
\hline$\lambda_{1}$ & $\theta_{\lambda_{1}}$ & $\lambda_{2}$ & $\theta_{\lambda_{2}}$ & $\mu_{1}$ & $\theta_{\mu_{1}}$ & $\mu_{2}$ & $\theta_{\mu_{2}}$ & $\alpha$ & $\theta_{\alpha}$ & $\varphi$ & $\theta_{\varphi}$ \\
\hline 0.1 & -0.8022 & 0.1 & -0.9521 & 0.1 & 0.05685 & 0.1 & 0.05852 & 0.1 & 0.09274 & 0.1 & -0.1393 \\
0.2 & -0.6435 & 0.2 & -0.9029 & 0.2 & 0.06709 & 0.2 & 0.07639 & 0.2 & 0.12014 & 0.2 & -0.1386 \\
0.3 & -0.5267 & 0.3 & -0.8547 & 0.3 & 0.06637 & 0.3 & 0.08069 & 0.3 & 0.1269 & 0.3 & -0.1242 \\
0.4 & -0.4422 & 0.4 & -0.8087 & 0.4 & 0.0627 & 0.4 & 0.07986 & 0.4 & 0.1258 & 0.4 & -0.1101 \\
0.5 & -0.3804 & 0.5 & -0.7657 & 0.5 & 0.05845 & 0.5 & 0.07705 & 0.5 & 0.12165 & 0.5 & -0.0982 \\
\hline
\end{tabular}

TABLE 6: Relative sensitivity of MTTFF when $r_{1}<r_{2}$.

\begin{tabular}{ccccccccccccc}
\hline$\lambda_{1}$ & $\theta_{\lambda_{1}}$ & $\lambda_{2}$ & $\theta_{\lambda_{2}}$ & $\mu_{1}$ & $\theta_{\mu_{1}}$ & $\mu_{2}$ & $\theta_{\mu_{2}}$ & $\alpha$ & $\theta_{\alpha}$ & $\varphi$ & $\theta_{\varphi}$ \\
\hline 0.1 & -0.9434 & 0.1 & -0.8249 & 0.1 & 0.0615 & 0.1 & 0.0635 & 0.1 & 0.1006 & 0.1 & -0.1492 \\
0.2 & -0.886 & 0.2 & -0.6775 & 0.2 & 0.0775 & 0.2 & 0.0756 & 0.2 & 0.1303 & 0.2 & -0.148 \\
0.3 & -0.8309 & 0.3 & -0.5644 & 0.3 & 0.07996 & 0.3 & 0.0751 & 0.3 & 0.1373 & 0.3 & -0.1326 \\
0.4 & -0.7791 & 0.4 & -0.4802 & 0.4 & 0.0778 & 0.4 & 0.07134 & 0.4 & 0.1358 & 0.4 & -0.1175 \\
0.5 & -0.7313 & 0.5 & -0.4171 & 0.5 & 0.0741 & 0.5 & 0.06669 & 0.5 & 0.1309 & 0.5 & -0.1046 \\
\hline
\end{tabular}

TABle 7: Sensitivity of $\operatorname{Av}(\infty)$ when $r_{1}=r_{2}$.

\begin{tabular}{cccccccccccc}
\hline$\lambda_{1}$ & $\sigma_{\lambda_{1}}$ & $\lambda_{2}$ & $\sigma_{\lambda_{2}}$ & $\mu_{1}$ & $\sigma_{\mu_{1}}$ & $\mu_{2}$ & $\sigma_{\mu_{2}}$ & $\alpha$ & $\sigma_{\alpha}$ & $\varphi$ & $\sigma_{\varphi}$ \\
\hline 0.1 & -0.866 & 0.1 & -0.9143 & 0.1 & 1.5266 & 0.1 & 1.5465 & 0.1 & 2.2148 & 0.1 & -0.7801 \\
0.2 & -0.5579 & 0.2 & -0.5931 & 0.2 & 0.3709 & 0.2 & 0.3908 & 0.2 & 0.6829 & 0.2 & -0.328 \\
0.3 & -0.3796 & 0.3 & -0.406 & 0.3 & 0.1342 & 0.3 & 0.1465 & 0.3 & 0.3047 & 0.3 & -0.1788 \\
0.4 & -0.2691 & 0.4 & -0.289 & 0.4 & 0.05946 & 0.4 & 0.06741 & 0.4 & 0.1651 & 0.4 & -0.1125 \\
0.5 & -0.1969 & 0.5 & -0.2123 & 0.5 & 0.02968 & 0.5 & 0.03507 & 0.5 & 0.1009 & 0.5 & -0.0773 \\
\hline
\end{tabular}

Table 8: Sensitivity of $\operatorname{Av}(\infty)$ when $r_{1}>r_{2}$.

\begin{tabular}{cccccccccccc}
\hline$\lambda_{1}$ & $\sigma_{\lambda_{1}}$ & $\lambda_{2}$ & $\sigma_{\lambda_{2}}$ & $\mu_{1}$ & $\sigma_{\mu_{1}}$ & $\mu_{2}$ & $\sigma_{\mu_{2}}$ & $\alpha$ & $\sigma_{\alpha}$ & $\varphi$ & $\sigma_{\varphi}$ \\
\hline 0.1 & -1.116 & 0.1 & -1.0266 & 0.1 & 1.648 & 0.1 & 1.659 & 0.1 & 2.174 & 0.1 & -0.8694 \\
0.2 & -0.5382 & 0.2 & -0.6912 & 0.2 & 0.4158 & 0.2 & 0.4917 & 0.2 & 0.703 & 0.2 & -0.3966 \\
0.3 & -0.2957 & 0.3 & -0.4911 & 0.3 & 0.1438 & 0.3 & 0.2022 & 0.3 & 0.32301 & 0.3 & -0.2261 \\
0.4 & -0.1766 & 0.4 & -0.3624 & 0.4 & 0.05737 & 0.4 & 0.09857 & 0.4 & 0.1786 & 0.4 & -0.1459 \\
0.5 & -0.1117 & 0.5 & -0.2755 & 0.5 & 0.02385 & 0.5 & 0.05333 & 0.5 & 0.1108 & 0.5 & -0.1018 \\
\hline
\end{tabular}

Table 9: Sensitivity of $\operatorname{Av}(\infty)$ when $r_{1}<r_{2}$.

\begin{tabular}{cccccccccccc}
\hline$\lambda_{1}$ & $\sigma_{\lambda_{1}}$ & $\lambda_{2}$ & $\sigma_{\lambda_{2}}$ & $\mu_{1}$ & $\sigma_{\mu_{1}}$ & $\mu_{2}$ & $\sigma_{\mu_{2}}$ & $\alpha$ & $\sigma_{\alpha}$ & $\varphi$ & $\sigma_{\varphi}$ \\
\hline 0.1 & -0.9978 & 0.1 & -1.1862 & 0.1 & 1.653 & 0.1 & 1.664 & 0.1 & 2.224 & 0.1 & -0.9014 \\
0.2 & -0.6701 & 0.2 & -0.5786 & 0.2 & 0.4752 & 0.2 & 0.4392 & 0.2 & 0.7319 & 0.2 & -0.4088 \\
0.3 & -0.4738 & 0.3 & -0.3198 & 0.3 & 0.1879 & 0.3 & 0.159 & 0.3 & 0.339 & 0.3 & -0.2323 \\
0.4 & -0.3482 & 0.4 & -0.1913 & 0.4 & 0.08789 & 0.4 & 0.06727 & 0.4 & 0.1878 & 0.4 & -0.1496 \\
0.5 & -0.2637 & 0.5 & -0.1206 & 0.5 & 0.04537 & 0.5 & 0.03065 & 0.5 & 0.1166 & 0.5 & -0.1043 \\
\hline
\end{tabular}


TABLE 10: Relative sensitivity of $\operatorname{Av}(\infty)$ when $r_{1}=r_{2}$.

\begin{tabular}{cccccccccccc}
\hline$\lambda_{1}$ & $\prod_{\lambda_{1}}$ & $\lambda_{2}$ & $\prod_{\lambda_{2}}$ & $\mu_{1}$ & $\prod_{\mu_{1}}$ & $\mu_{2}$ & $\prod_{\mu_{2}}$ & $\alpha$ & $\prod_{\alpha}$ & $\varphi$ & $\prod_{\varphi}$ \\
\hline 0.1 & -0.09758 & 0.1 & -0.1037 & 0.1 & 0.2618 & 0.1 & 0.2667 & 0.1 & 0.3167 & 0.1 & -0.1008 \\
0.2 & -0.137 & 0.2 & -0.1469 & 0.2 & 0.1123 & 0.2 & 0.1184 & 0.2 & 0.1658 & 0.2 & -0.0904 \\
0.3 & -0.1476 & 0.3 & -0.1606 & 0.3 & 0.0589 & 0.3 & 0.0643 & 0.3 & 0.1051 & 0.3 & -0.0767 \\
0.4 & -0.1456 & 0.4 & -0.1598 & 0.4 & 0.0344 & 0.4 & 0.03882 & 0.4 & 0.07398 & 0.4 & -0.0657 \\
0.5 & -0.1375 & 0.5 & -0.1519 & 0.5 & 0.02128 & 0.5 & 0.0251 & 0.5 & 0.05579 & 0.5 & -0.0571 \\
\hline
\end{tabular}

TABLE 11: Relative sensitivity of $\operatorname{Av}(\infty)$ when $r_{1}>r_{2}$.

\begin{tabular}{ccccccccccccc}
\hline$\lambda_{1}$ & $\prod_{\lambda_{1}}$ & $\lambda_{2}$ & $\prod_{\lambda_{2}}$ & $\mu_{1}$ & $\prod_{\mu_{1}}$ & $\mu_{2}$ & $\prod_{\mu_{2}}$ & $\alpha$ & $\prod_{\alpha}$ & $\varphi$ & $\prod_{\varphi}$ \\
\hline 0.1 & -0.1365 & 0.1 & -0.1181 & 0.1 & 0.3126 & 0.1 & 0.3269 & 0.1 & 0.3328 & 0.1 & -0.117 \\
0.2 & -0.1456 & 0.2 & -0.1762 & 0.2 & 0.1356 & 0.2 & 0.1639 & 0.2 & 0.1801 & 0.2 & -0.1161 \\
0.3 & -0.127 & 0.3 & -0.2026 & 0.3 & 0.06751 & 0.3 & 0.09598 & 0.3 & 0.1173 & 0.3 & -0.1038 \\
0.4 & -0.1045 & 0.4 & -0.2117 & 0.4 & 0.03542 & 0.4 & 0.06101 & 0.4 & 0.08403 & 0.4 & -0.0918 \\
0.5 & -0.0843 & 0.5 & -0.2108 & 0.5 & 0.01829 & 0.5 & 0.04081 & 0.5 & 0.06405 & 0.5 & -0.0817 \\
\hline
\end{tabular}

TABle 12: Relative sensitivity of $\operatorname{Av}(\infty)$ when $r_{1}<r_{2}$.

\begin{tabular}{cccccccccccc}
\hline$\lambda_{1}$ & $\prod_{\lambda_{1}}$ & $\lambda_{2}$ & $\prod_{\lambda_{2}}$ & $\mu_{1}$ & $\prod_{\mu_{1}}$ & $\mu_{2}$ & $\prod_{\mu_{2}}$ & $\alpha$ & $\prod_{\alpha}$ & $\varphi$ & $\prod_{\varphi}$ \\
\hline 0.1 & -0.115 & 0.1 & -0.1469 & 0.1 & 0.3329 & 0.1 & 0.3261 & 0.1 & 0.3471 & 0.1 & -0.1231 \\
0.2 & -0.1694 & 0.2 & -0.1598 & 0.2 & 0.1617 & 0.2 & 0.1468 & 0.2 & 0.1902 & 0.2 & -0.1217 \\
0.3 & -0.1934 & 0.3 & -0.1409 & 0.3 & 0.09122 & 0.3 & 0.07618 & 0.3 & 0.1239 & 0.3 & -0.1088 \\
0.4 & -0.2006 & 0.4 & -0.1167 & 0.4 & 0.05571 & 0.4 & 0.04229 & 0.4 & 0.08889 & 0.4 & -0.0961 \\
0.5 & -0.1986 & 0.5 & -0.0941 & 0.5 & 0.03559 & 0.5 & 0.02391 & 0.5 & 0.06773 & 0.5 & -0.0855 \\
\hline
\end{tabular}

From these tables, we can see that the order of relative sensitivity on MTTFF affected by each system parameter can be ranked as follows:

(1) In the case $r_{1}=r_{2}$, we get $\lambda_{1}>\lambda_{2}>\varphi>\alpha>\mu_{2}>\mu_{1}$

(2) In the case $r_{1}>r_{2}$, we get $\lambda_{2}>\lambda_{1}>\varphi>\alpha>\mu_{2}>\mu_{1}$

(3) In the case $r_{1}<r_{2}$, we get $\lambda_{1}>\lambda_{2}>\varphi>\alpha>\mu_{2}>\mu_{1}$

The sensitivity for $A(\infty)$ can be obtained in Tables 7-9. In these tables, we can see that the order of sensitivity on steady-state availability affected by each system parameter can be ranked as follows:

(1) In the case $r_{1}=r_{2}$, we get $\alpha>\mu_{2}>\mu_{1}>\lambda_{2}>\lambda_{1}>\varphi$

(2) In the case $r_{1}>r_{2}$, we get $\alpha>\mu_{2}>\mu_{1}>\lambda_{1}>\lambda_{2}>\varphi$

(3) In the case $r_{1}<r_{2}$, we get $\alpha>\mu_{2}>\mu_{1}>\lambda_{2}>\lambda_{1}>\varphi$

Finally, the results of the relative sensitivity on steadystate availability affected by each system parameter are listed in Tables 10-12. The order of relative sensitivity on steadystate availability affected by each system parameter can be ranked as follows:

(1) In the case $r_{1}=r_{2}$, we get $\alpha>\mu_{2} \simeq \mu_{1}>\lambda_{2}>\varphi>\lambda_{1}$

(2) In the case $r_{1}>r_{2}$, we get $\alpha>\mu_{2}>\mu_{1}>\lambda_{1}>\lambda_{2}>\varphi$

(3) In the case $r_{1}<r_{2}$, we get $\alpha>\mu_{1}>\mu_{2}>\lambda_{2}>\varphi>\lambda_{1}$

\section{Conclusion}

This article studies the reliability measurements and sensitivity analysis of a system consisting of two nonidentical parallel units and a single repairman. The repairman might take a vacation or not at the beginning of the system operation, and the active units might be attacked by successive shocks. Such a system can be considered as an extension of repairable systems in the engineering industry. It is therefore difficult to analyze because many random variables with general distributions are involved. The expressions for steady-state availability and mean time to system failure with general repair time are derived from Laplace transform equations. Both sensitivity analysis and relative sensitivity analysis were calculated for three different cases of this system; the results are shown in Tables 1-12. Finally, the numerical results explain the relationship between the derived reliability measurements and the system parameters. From the numerical results and graphs, we deduce the following:

(1) The steady-state availability increases when $r_{1}=r_{2}$

(2) The mean to system failure increases when $r_{1}=r_{2}$

\section{Notations}

$h(t), H(t):$

$v_{1}(t), V_{1}(t)$ :

$v_{2}(t), V_{2}(t)$ :

p.d.f. and c.d.f. of the repair time.

p.d.f. and c.d.f. of the vacation time of a repairman.

p.d.f. and c.d.f. of the recall time of a repairman

$P_{i}(t, x), Q_{i}(t, x): \quad$ p.d.f. and c.d.f. of the system are in state $i=2,4$ at period $t$ and have an elapsed repair time of $x$ for unit $A$

$P_{i}(t, y), Q_{i}(t, y): \quad$ p.d.f. and c.d.f. of the system are in state $i=1,8$ at period $t$ and have an elapsed repair time of $y$ for unit $B$ 
$P_{o}(t, u), Q_{o}(t, u):$ p.d.f. and c.d.f. of the system are in state $s_{o}$ at period $t$ and have an elapsed vacation time of $u$

$P_{i}(t, z), Q_{i}(t, x): \quad$ p.d.f. and c.d.f. of the system are in state $i=5,6,7$ at period $t$ and have recalling time of $z$

$\varphi(u)$ Vacation time distribution function

$\mu_{1}(x): \quad$ The repair rate of unit $\mathrm{A}$

$\mu_{2}(y): \quad$ The repair rate of unit $\mathrm{B}$

$\alpha(z): \quad$ Call time distribution function

$h^{*}(s): \quad$ L.T (Laplace transform) $h(\mathrm{t})$

$p_{i}(\mathrm{t})$ : $\quad$ The probability of the system to be in state $i$ at time $t$.

\section{Data Availability}

The data used to support the study were obtained from previous research.

\section{Disclosure}

A preprint has previously been published [17].

\section{Conflicts of Interest}

The authors declare that they have no conflicts of interest.

\section{Acknowledgments}

This research was funded by the Deanship of Scientific Research (DSR) at King Abdulaziz University, Jeddah, under Grant No. G:131-662-1439. The authors, therefore, acknowledge DSR with thanks for technical and financial support.

\section{References}

[1] L. Vonta and M. Ram, Reliability Engineering: Theory and Applications, Taylor \& Francis Group, UK, 2021.

[2] Z. Lin, D. Cevasco, and M. A. Collu, "Methodology to develop reduced-order models to support the operation and maintenance of offshore wind turbines," Applied Energy, vol. 259, Article ID 114228, 2021.

[3] Z. Ren, A. S. Verma, Y. Li, J. J. E. Teuwen, and Z. Jiang, "Offshore wind turbine operations and maintenance: a stateof-the-art review," Renewable and Sustainable Energy Reviews, vol. 144, Article ID 110886, 2021.

[4] Y. Lam and Y. L. Zhang, "A shock model for the maintenance problem of a repairable system," Computers \& Operations Research, vol. 31, no. 11, pp. 1807-1820, 2004.

[5] Z. Li and P. Zhao, "Reliability analysis on the \$ldelta\$-Shock model of complex systems," IEEE Transactions on Reliability, vol. 56, no. 2, pp. 340-348, 2007.

[6] M. S. El-Sherbeny, "Stochastic behavior of a two-unit cold standby redundant system under Poisson shocks," Arabian Journal for Science and Engineering, vol. 42, no. 7, pp. 30433053, 2017.

[7] M. A. W. Mahmoud, A. M. Rashad, and Z. M. Hussien, "Stochastic analysis of a repairable cold standby system Attacked by Poisson shocks considering inspection and post repair," International Journal of Computer Application, vol. 132, no. 11, pp. 33-40, 2015.
[8] Q. Wu and S. Wu, "Reliability analysis of two-unit cold standby repairable systems under Poisson shocks," Applied Mathematics and Computation, vol. 218, no. 1, pp. 171-182, 2011.

[9] J.-C. Ke and C.-H. Lin, "A Markov repairable system involving an imperfect service station with multiple vacations," Asia Pacific Journal of Operational Research, vol. 22, no. 04, pp. 555-582, 2005.

[10] R. B. Liu, Y. H. Tang, and C. Y. Luo, "A new kind of n-unit series repairable system and its reliability analysis," Mathematica Applicata, vol. 20, no. 1, pp. 164-170, 2007.

[11] L. M. Hu, J. D. Li, and W. M. Fang, "Reliability analysis of an $\mathrm{n}$-component series system with $\mathrm{m}$ failure modes and vacation," ICIC Express Letters, vol. 2, pp. 53-58, 2008.

[12] L. M. Hu and J. D. Li, "Reliability analysis of a three-unit system with vacation and priority," ICIC Express Letters, vol. 3, pp. 171-176, 2009.

[13] Y. Chen, X. Meng, and S. Chen, "Reliability analysis of a cold standby system with imperfect repair and under Poisson shocks," Mathematical Problems in Engineering, vol. 2014, Article ID 507846, 11 pages, 2014.

[14] Y. Levy and U. Yechiali, "Utilization of idle time in anM/G/1 queueing system M/G/1 queue," Management Science, vol. 22, no. 2, pp. 202-211, 1975.

[15] N. S. Tian and Z. G. Zhang, Vacation Queueing Models-Theory and Applications, Springer-Verlag, New York, 2006.

[16] J.-C. Ke and K.-H. Wang, "Vacation policies for machine repair problem with two type spares," Applied Mathematical Modelling, vol. 31, no. 5, pp. 880-894, 2007.

[17] S. Mohamed, El-Sherbeny, and M. Zienab, "Stochastic Behavior of a Two-Unit Parallel System with Dissimilar Units and Optional Vacations under Poisson Shocks," 4 February 2021, https://www.preprints.org/manuscript/202102.0138/ v1,\%20Received:\%203\%20February\%202021\%20/\% 20Approved. 